\title{
High overexpression of fatty acid synthase is associated with poor survival in Chinese patients with gastric carcinoma
}

\author{
WENMIN HOU ${ }^{1,2^{*}}$, MAOGUI FEI ${ }^{2 *}$, XIA QIN $^{3,5}$, XUEHUA ZHU ${ }^{4}$, JOEL GRESHOCK $^{5}$, PING LIU $^{3}$, \\ YUANFENG ZHOU ${ }^{3,5}$, HUI WANG ${ }^{2}$, BANG-CE YE ${ }^{1}$ and CRYSTAL YING QIN ${ }^{3,5}$ \\ ${ }^{1}$ Laboratory of Biosystems and Microanalysis, State Key Laboratory of Bioreactor Engineering, \\ East China University of Science and Technology; ${ }^{2}$ Key Laboratory of Nutrition and Metabolism, \\ Institute for Nutritional Sciences, Shanghai Institutes for Biological Sciences, Chinese Academy of Sciences, \\ Graduate School of the Chinese Academy of Sciences; ${ }^{3}$ Department of Oncology, GlaxoSmithKline Research and \\ Development Center; ${ }^{4}$ Shanghai Key Laboratory of Signaling and Disease Research, School of Life Science and Technology, \\ Tongji University, Shanghai, P.R. China; ${ }^{5}$ Cancer Research, GlaxoSmithKline, Collegeville, PA, USA
}

Received February 6, 2012; Accepted September 19, 2012

DOI: $10.3892 / \mathrm{etm} .2012 .727$

\begin{abstract}
Fatty acid synthase (FAS) is the key enzyme regulating de novo biosynthesis of fatty acids. FAS overexpression has been found in many types of tumors and is associated with poor survival. However, the expression of FAS and its relationship with prognosis in Chinese patients with gastric carcinoma are still unknown. Therefore, in this study, we examined the expression of FAS using tissue microarrays and determined its correlation with clinicopathological characteristics and prognosis of gastric carcinoma in Chinese patients. FAS overexpression was graded as $\mathrm{S}$ (T/A) $<1, \geq 1$ to $<2, \geq 2$ to $<3$ or $\geq 3$ in 35 (38.9\%), 20 (22.2\%), 9 (10\%) and $26(28.9 \%)$ patients, respectively. High FAS overexpression [S (T/A) $\geq 3$ ] was significantly correlated with poor prognosis (log-rank test, $\mathrm{P}=0.0078)$ and with decreased 3 -year survival rate $\left(\chi^{2}\right.$ test, $\mathrm{P}=0.0023)$. FAS overexpression was not significantly associated with other clinicopathological characteristics. In conclusion, our results suggest that FAS expression might be a potential prognostic marker for gastric carcinoma in Chinese patients.
\end{abstract}

Correspondence to: Dr Bang-Ce Ye, Laboratory of Biosystems and Microanalysis, State Key Laboratory of Bioreactor Engineering, East China University of Science and Technology, 130 Meilong Road, Shanghai 200237, P.R. China

E-mail: bcye@ecust.edu.cn

Dr Crystal Ying Qin, Department of Oncology, GlaxoSmithKline Research and Development Center, 917 Halei Road, Zhangjiang Hi-Tech Park, Shanghai 201203, P.R. China

E-mail: crystal.y.qin@gsk.com

"Contributed equally

Key words: fatty acid synthase, gastric cancer, tissue microarray, prognostic marker

\section{Introduction}

Fatty acids (FAs) are biological molecules with important physiological roles in energy storage, membrane formation and protein acylation (1). Animals acquire FAs from the diet and via de novo biosynthesis (2). In the latter, FAs are predominantly generated by a 250 - to $270-\mathrm{kDa}$ multifunctional and homodimeric enzyme, fatty acid synthase (FAS). Long-chain FAs, the main product of FAS, are derived from acetyl-CoA, malonylCoA and NADPH (3).FAs are essential constituents of biological membranes and are important substrates in energy metabolism. Although the mechanisms responsible for FAS overexpression in tumors are not fully understood, the PTEN/PI3K/AKT and RAS/RAF/MAPK/ERK1/2 pathways are known to regulate FAS expression $(3,4)$, and these pathways are often hyperactive in tumors. Notably, in the LNCaP prostate cancer cell line, pharmacological inhibition of PI3K or reintroduction of wild-type PTEN was found to reduce FAS expression (4).

Most tissues, except for the liver, adipose tissue, cycling endometrium (5), fetal lungs (6), lactating breast $(7,8)$ and embryos $(3,9)$ utilize dietary FAs to build new structural lipids. Therefore, FAS is expressed at low levels in most normal tissues. By contrast, in cancer tissues, the FA supply is highly dependent on de novo biosynthesis via FAS. Indeed, several studies have shown that FAS is overexpressed in many cancers, including breast $(10,11)$, prostate $(12,13)$, ovarian $(14)$ and colorectal carcinomas $(15,16)$. Furthermore, high FAS expression is associated with advanced clinical stage, poor differentiation and poor prognosis of breast (10), prostate (17) and ovarian carcinomas (15). Downregulation of FAS by RNAi was found to inhibit growth and apoptosis in LnCaP cells but not in normal fibroblasts (18). Furthermore, pharmacological or RNAi-mediated downregulation of FAS significantly sensitized the responsiveness of breast cancer cell lines (SK-Br3, MCF-7 and MDA-MB-231) to paclitaxel or vinorelbine $(19,20)$. These results indicate that FAS is an important prognostic factor in certain types of cancers and may represent a potential therapeutic target for cancer chemotherapy. 
However, FAS expression in gastric carcinoma, one of the most prevalent malignant tumors worldwide, particularly in China, has not been established. To date, few clinical studies have determined FAS expression in gastric carcinoma or compared its expression with that in non-neoplastic adjacent tissue $(21,22)$. Since FAS expression varies at different ages and clinical circumstances, determining FAS expression in tumor tissue alone is insufficient to clarify the prognostic relevance of FAS expression in cancer. Therefore, to provide insight into the clinical relevance of FAS, we examined FAS expression in gastric carcinoma and paired adjacent normal tissue samples collected from 90 Chinese patients. We analyzed the associations between FAS expression and clinicopathological characteristics, such as age, gender, histological grade, American Joint Committee on Cancer (AJCC) tumor stage, metastasis and tumor size, as well as molecular markers, such as the loss of PTEN and pERK1/2 expression. Finally, we determined the effects of FAS expression on prognosis.

\section{Materials and methods}

Patients and tissue samples. Ninety patients with gastric carcinoma who underwent surgery between 2007 and 2008 were enrolled in this study. None of the patients had received any treatment before surgery. We obtained complete clinicopathological information for all patients, including age, gender, tumor size, histological grade, AJCC tumor stage, depth of invasion, lymph node metastasis and distant metastasis. All of the patients included in this study had adenocarcinoma. The median age of the patients at the time of diagnosis was 65.5 years (range 34-83 years). The histological grade of the tumor was evaluated based on the degree of tumor differentiation, tumor necrosis and mitotic count, according to the criteria of Enzinger and Weiss (23). Follow-up time was calculated as the time from initial surgery to the death of the patient due to the primary tumor or the date of last contact. Tumor tissue and paired adjacent normal tissue samples were obtained at surgery. All tissues were dissected in the operating room, immediately frozen, and stored at $-80^{\circ} \mathrm{C}$. Informed consent for use of tissue samples in future molecular studies was obtained from each patient. This study was approved by the Ethics Committee of the Third Xiangya Hospital, Central South University (Hunan, China). Clinical and treatment information was extracted by chart review carried out by the surgeon with approval from our institutional review board.

Tissue microarray (TMA) preparation. Core needle biopsies (1.5 $\mathrm{mm}$ diameter) were extracted from paraffin-embedded tissue samples, and mounted into a recipient paraffin block using a dedicated tissue array instrument (Beecher Instruments, Sun Prairie, WI, USA). Then, $4-\mu \mathrm{m}$-thick sections of the TMA were cut, transferred to glass slides and stained with hematoxylin and eosin.

Immunohistochemistry. TMA sections (1.5-mm diameter; $4-\mu \mathrm{m}$ thick) from archival, formalin-fixed, paraffin-embedded tissue specimens were mounted on poly-L-lysine (Muto Chemicals, Tokyo, Japan)-coated slides. The TMA sections were deparaffinized in xylene for $15 \mathrm{~min}$, rehydrated in an ethanol gradient and heated at $95^{\circ} \mathrm{C}$ for $5 \mathrm{~min}$ in $10 \mathrm{mM}$ sodium citrate buffer
Table I. TMA clinical information.

\begin{tabular}{lc} 
Characteristics & \\
\hline Gender, $\mathrm{n}(\%)$ & $67(74.4)$ \\
$\quad$ Male & $23(25.6)$ \\
$\quad$ Female & \\
Age (years) & $65(34-83)$ \\
$\quad$ Median (range) & \\
Histological type, $\mathrm{n}(\%)$ & $90(100.0)$ \\
$\quad$ Adenocacinoma & $0 \quad(0.0)$ \\
Others & \\
Presentation, n (\%) & $90(100.0)$ \\
Initial & $0 \quad(0.0)$ \\
Recurrent & \\
Size (cm) & $5.75(0-20)$ \\
$\quad$ Median (range) & \\
Grade, $\mathrm{n}(\%)$ & $1 \quad(1.1)$ \\
I & $23(25.6)$ \\
II & $66(73.3)$ \\
III & \\
Metastasis, $n(\%)$ & $20(22.2)$ \\
$\quad$ Negative & $70(77.8)$ \\
Positive &
\end{tabular}

(pH 6.0) in a microwave oven for antigen retrieval. Endogenous peroxidase was inactivated by incubating the sections in $3 \%$ $\mathrm{H}_{2} \mathrm{O}_{2}$ for $15 \mathrm{~min}$ at room temperature. The sections were blocked in $3 \%$ normal donkey serum and incubated at $4^{\circ} \mathrm{C}$ overnight with monoclonal anti-FAS antibody (dilution, 1:50; no. 3180S, Cell Signaling Technology, Danvers, MA, USA), antipERK1/2 antibody (dilution, 1:1000; no. 4370, Cell Signaling Technology) and anti-PTEN antibody (dilution, 1:50; no. 9559C, Cell Signaling Technology). Finally, the sections were stained with horseradish peroxidase-conjugated donkey antirabbit IgG $(\mathrm{H}+\mathrm{L})$ secondary antibody (711-035-152, Jackson ImmunoResearch Europe, Newmarket, UK). Signal detection was carried out using a Dako signaling amplification system (K346811; Dako, Glostrup, Denmark). The TMA sections were counterstained with hematoxylin, dehydrated, and mounted.

TMA score. Immunohistochemistry was scored based on staining intensity and the percentage of positive cells. The staining intensity was scored as follows: 0 , negative; 1 , weak; 2 , moderate; and 3, high intensity. The immunoreactive score was calculated as staining intensity score $\mathrm{x}$ percentage of FAS-positive cells. We also calculated S (T/A), immunoreactive score of tumor tissue/immunoreactive score of paired adjacent normal tissue, as an index for the difference in expression between tumor and normal tissue. FAS immunostaining was analyzed under a microscope (Nikon Eclipse E600) and estimated independently by two pathologists.

Statistical analysis. Statistical analyses were performed using GraphPad Prism 5 (GraphPad Software Inc., San Diego, CA, 
A

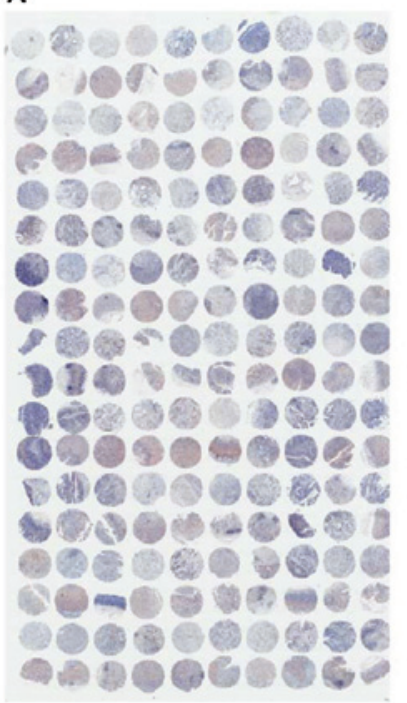

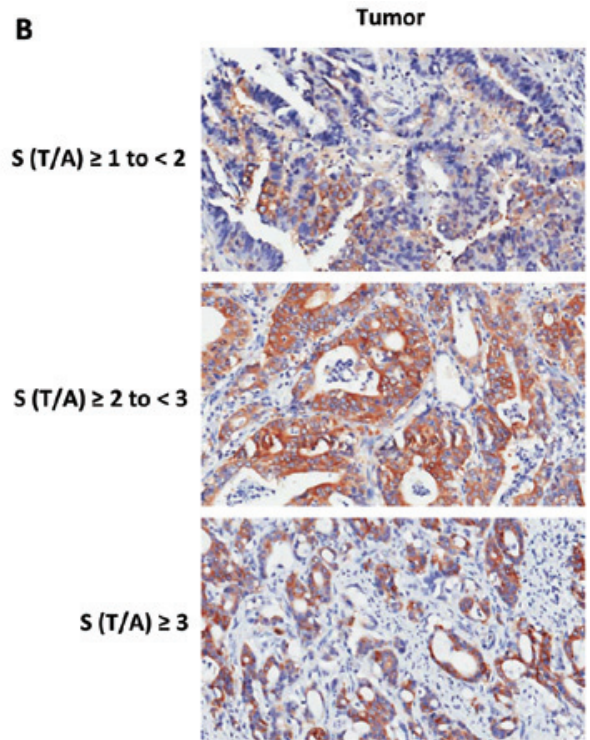

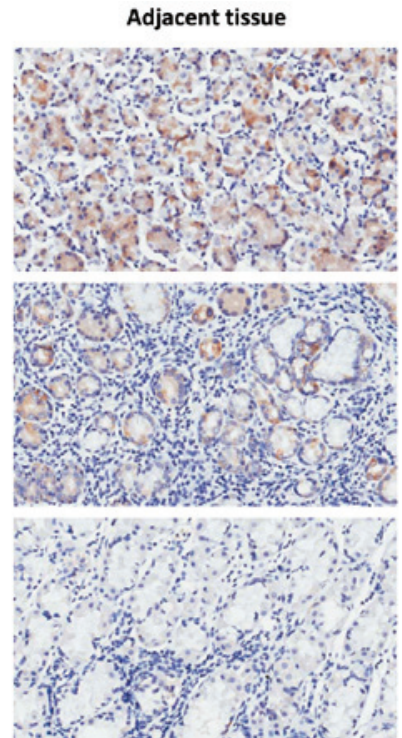

Figure 1. Immunohistochemical expression of FAS in gastric carcinomas. (A) Representative tissue microarray. The odd and even lines represent tumor and adjacent normal tissue samples, respectively. (B) Representative cases of immunohistochemical expression of FAS for S (T/A) $\geq 1$ to $<2$, $\mathrm{S}$ (T/A) $\geq 2$ to $<3$ and $\mathrm{S}(\mathrm{T} / \mathrm{A}) \geq 3$. $\mathrm{S}(\mathrm{T} / \mathrm{A})=$ immunohistochemistry score (tumor)/immunohistochemistry score (adjacent tissue). Immunohistochemistry score $=$ staining intensity $\mathrm{x}$ percent of FAS-positive cells. Staining intensity was scored as 0, negative; 1, weak; 2, moderate; and 3, high.

A

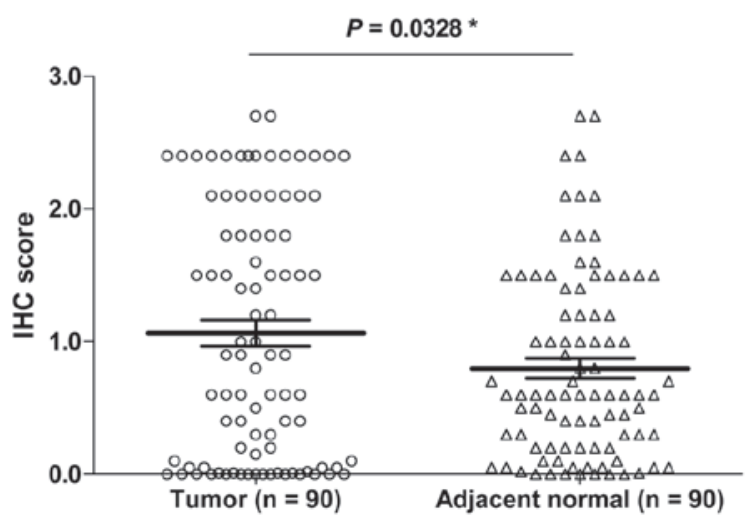

B

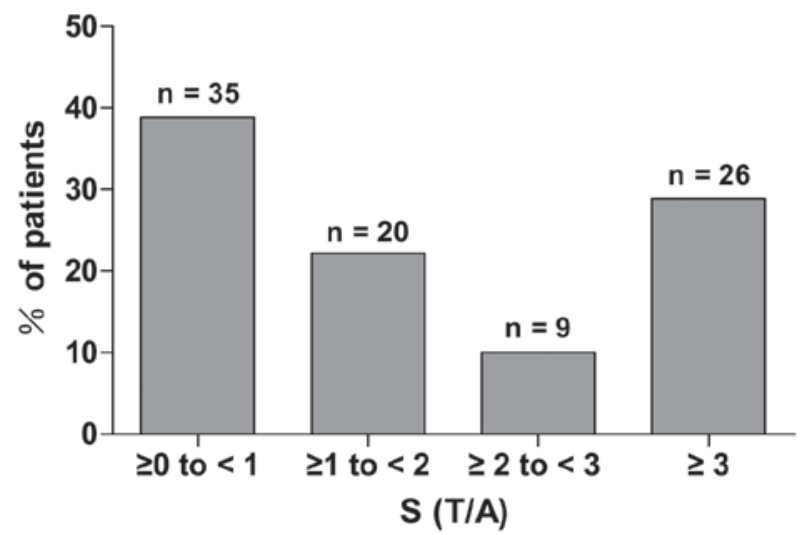

Figure 2. (A) Scatter plot showing the distribution of the immunohistochemical (IHC) score for FAS expression in gastric tumor tissues and adjacent normal tissues. The mean IHC scores for FAS expression in tumors and adjacent tissues are $1.065 \pm 0.099$ and $0.7981 \pm 0.074$, respectively. The P-value was determined by an unpaired test. (B) Distribution of FAS expression defined as S (T/A). Overall, 38.9\% (35/90), 22.2\% (20/90), 10\% (9/90) and 28.9\% (26/90) of the carcinoma tissue specimens were classified as $\mathrm{S}$ (T/A) $\geq 0$ to $<1, \mathrm{~S}$ (T/A) $\geq 1$ to $<2, \mathrm{~S}$ (T/A) $\geq 2$ to $<3$ and $\mathrm{S}$ (T/A) $\geq 3$, respectively.

USA) for Windows. The Student's t-test was used to compare FAS expression between cancer tissue and adjacent normal tissues. Contingency table analysis and $\chi^{2}$ tests were used to investigate the relationship between FAS expression and clinical variables. For outcomes with a small number of cases, Fisher's exact test was used. Survival was estimated using the Kaplan-Meier method, and differences in survival curves were determined using the log-rank test. Values of $\mathrm{P}<0.05$ were regarded as statistically significant.

\section{Results}

Clinicopathological characteristics. Table I summarizes the clinicopathological characteristics data of the patients with previously untreated gastric carcinoma. The study cohort included 67 males and 23 females, ranging in age from 34 to 83 years (median age, 65 years). The histological type of all patients was adenocarcinoma. Tumor size ranged from 0 to $20 \mathrm{~cm}$ with a mean size of $6.17 \mathrm{~cm}$ and a median size of $5.75 \mathrm{~cm}$. The tumors were classified as grade I in 1 patient, grade II in 23 patients and grade III in 66 patients. The median and mean duration of follow-up was 35 and 31.75 months, respectively, ranging from 1 to 51 months. Most of the patients $(77.8 \%, 70 / 90)$ developed distant or regional lymph node metastasis.

FAS expression. We determined FAS expression in all 90 tumor tissue and paired adjacent normal tissue samples by 
Table II. FAS expression and clinicopathological factors of the gastric carcinoma patients.

\begin{tabular}{|c|c|c|c|c|c|c|c|}
\hline & $\mathrm{S}(\mathrm{T} / \mathrm{A})<1$ & $\mathrm{~S}(\mathrm{~T} / \mathrm{A}) \geq 1$ & ${ }^{\text {ap}} \mathrm{P}$-value & $\mathrm{S}(\mathrm{T} / \mathrm{A}) \geq 2$ & ${ }^{b} \mathrm{P}$-value & $\mathrm{S}(\mathrm{T} / \mathrm{A}) \geq 3$ & ${ }^{\mathrm{c}} \mathrm{P}$-value \\
\hline \multicolumn{8}{|c|}{ Age (years), n (\%) } \\
\hline Median age & 65 & 65 & & 69 & & 65 & \\
\hline Range & $45-83$ & $34-83$ & & $41-81$ & & $41-81$ & \\
\hline$<60$ & $14(48.3)$ & $15(51.7)$ & 0.2078 & $7(24.1)$ & 0.0645 & $6(20.7)$ & 0.6086 \\
\hline$\geq 60$ & $21(34.4)$ & $40(65.6)$ & & $28(45.9)$ & & $20(32.8)$ & \\
\hline \multicolumn{8}{|l|}{ Gender, n (\%) } \\
\hline Female & $6(26.1)$ & $17(73.9)$ & 0.1444 & $12(52.2)$ & 0.1298 & $9(39.1)$ & 0.286 \\
\hline Male & $29(43.3)$ & $38(56.7)$ & & $23(34.3)$ & & $17(25.4)$ & \\
\hline \multicolumn{8}{|c|}{ Histological grade, n (\%) } \\
\hline I & $0 \quad(0.0)$ & $1(100.0)$ & 0.4328 & $0 \quad(0.0)$ & 0.4524 & $0 \quad(0.0)$ & 0.7564 \\
\hline II & $7(30.4)$ & $16(69.6)$ & & $11(47.8)$ & & $6(26.1)$ & \\
\hline III & $28(42.4)$ & $38(57.6)$ & & $24(36.4)$ & & $20(30.3)$ & \\
\hline \multicolumn{8}{|c|}{ AJCC tumor stage, n (\%) } \\
\hline I & $1(16.7)$ & $5(83.3)$ & 0.5244 & $2(33.3)$ & 0.4868 & $1(16.7)$ & 0.2082 \\
\hline II & $12(40.0)$ & $18(60.0)$ & & $11(36.7)$ & & $6(20.0)$ & \\
\hline III & $20(39.2)$ & $31(60.8)$ & & $22(43.1)$ & & $19(37.3)$ & \\
\hline IV & $2(66.7)$ & $1(33.3)$ & & $\begin{array}{ll}0 & (0.0)\end{array}$ & & $0 \quad(0.0)$ & \\
\hline \multicolumn{8}{|c|}{ Metastasis, n (\%) } \\
\hline Negative & $5 \quad(25)$ & $15(75.0)$ & 0.1485 & $8(40.0)$ & 0.908 & $5(25.0)$ & 0.6635 \\
\hline Positive & $30(42.9)$ & $40(57.1)$ & & $27(38.6)$ & & $21(30.0)$ & \\
\hline \multicolumn{8}{|c|}{ Tumor size $(\mathrm{cm}), \mathrm{n}(\%)$} \\
\hline$<5$ & $14(45.2)$ & $17(54.8)$ & 0.3763 & $11(35.5)$ & 0.631 & $7(22.6)$ & 0.3385 \\
\hline$\geq 5$ & $21(35.6)$ & $38(64.4)$ & & $24(40.7)$ & & $19(32.2)$ & \\
\hline \multicolumn{8}{|c|}{ PTEN (total), n (\%) } \\
\hline Negative & $12(36.4)$ & $21(63.6)$ & 0.8234 & $13(39.4)$ & 1.0 & $10(30.3)$ & 0.8145 \\
\hline Positive & $23(40.4)$ & $34(59.6)$ & & $22(38.6)$ & & $16(28.1)$ & \\
\hline \multicolumn{8}{|c|}{ PTEN (cytopasmic), n (\%) } \\
\hline Negative & $24(39.3)$ & $37(60.7)$ & 1.0 & $23(37.7)$ & 0.8184 & $16(26.2)$ & 0.4612 \\
\hline Positive & $11(37.9)$ & $18(62.1)$ & & $12(41.4)$ & & $10(36.7)$ & \\
\hline \multicolumn{8}{|c|}{ PTEN (nuclear), n (\%) } \\
\hline Negative & $14(35.0)$ & $26(65.0)$ & 0.5224 & $16(40.0)$ & 1.0 & $12(30.0)$ & 1.0 \\
\hline Positive & $21(42.0)$ & $29(58.0)$ & & $19(38.0)$ & & $14(28.0)$ & \\
\hline
\end{tabular}

P-value is determined by $\chi^{2}$ exact test, ${ }^{\text {a }} \mathrm{S}(\mathrm{T} / \mathrm{A})<1$ vs. $\mathrm{S}(\mathrm{T} / \mathrm{A}) \geq 1,{ }^{\mathrm{b}} \mathrm{S}(\mathrm{T} / \mathrm{A})<2$ vs. $\mathrm{S}(\mathrm{T} / \mathrm{A}) \geq 2,{ }^{\mathrm{c}} \mathrm{S}(\mathrm{T} / \mathrm{A})<3$ vs. $\mathrm{S}(\mathrm{T} / \mathrm{A}) \geq 3$.

TMA and immunohistochemistry. A representative TMA stained for FAS is shown in Fig. 1A. No signal was detected in the nuclei or on the cell membrane, indicating that FAS was mainly localized to the cytoplasm. The mean immunohistochemical score of FAS expression was significantly higher in tumor tissue than in adjacent normal tissue $(1.065 \pm 0.099 \mathrm{vs}$. $0.798 \pm 0.074, \mathrm{P}<0.05$, Fig. $2 \mathrm{~A})$. These data indicate that FAS was overexpressed in the cytoplasm in tumor tissue compared to the expression in the adjacent normal tissue. To investigate the difference in expression between tumor tissues and normal tissues, we calculated S (T/A) as described in Materials and methods. Representative tissue sections corresponding to $\mathrm{S}$ (T/A) $\geq 1$ to $<2$ (low), $\mathrm{S}$ (T/A) $\geq 2$ to $<3$ (medium) and $\mathrm{S}(\mathrm{T} / \mathrm{A}) \geq 3$ (high expression) are shown in Fig. 1B. Overall, 38.9\% (35/90),
$22.2 \%(20 / 90), 10 \%$ (9/90) and $28.9 \%$ (26/90) of the carcinoma tissue specimens were classified as $\mathrm{S}$ (T/A) $\geq 0$ to $<1, \mathrm{~S}$ (T/A) $\geq 1$ to $<2, \mathrm{~S}$ (T/A) $\geq 2$ to $<3$ and $\mathrm{S}$ (T/A) $\geq 3$, respectively (Fig. 2B).

Relationship between FAS overexpression and clinicopathological parameters. To investigate the association between FAS overexpression and clinicopathological parameters, three grades of FAS overexpression ( $\mathrm{S}(\mathrm{T} / \mathrm{A}) \geq 1, \geq 2$ and $\geq 3$ ) were established. FAS overexpression was not significantly associated with any of the clinicopathological variables recorded, including age, gender, grade, tumor size and lymph node metastasis (Table II). As several reports have shown that FAS expression is modulated by the PTEN/PI3K/AKT and RAS/ $\mathrm{RAF} / \mathrm{MEK} / \mathrm{ERK}$ pathways, we determined the expression of 
Table III. FAS expression and 3-year overall survival of the gastric carcinoma patients.

\begin{tabular}{lcccccc}
\hline $\begin{array}{l}\text { Overall } \\
\text { survival }\end{array}$ & $\mathrm{S}(\mathrm{T} / \mathrm{A})<1$ & $\mathrm{~S}(\mathrm{~T} / \mathrm{A}) \geq 1$ & $\mathrm{~S}(\mathrm{~T} / \mathrm{A})<2$ & $\mathrm{~S}(\mathrm{~T} / \mathrm{A}) \geq 2$ & $\mathrm{~S}(\mathrm{~T} / \mathrm{A})<3$ & $\mathrm{~S}(\mathrm{~T} / \mathrm{A}) \geq 3$ \\
\hline$\geq 3$ years & 18 & 26 & 29 & 15 & 38 & 6 \\
<3 years & 17 & 29 & 26 & 20 & 26 & 20 \\
P-value & \multicolumn{2}{r}{0.8292} & & 0.3939 & & 0.0023 \\
\hline
\end{tabular}

P-value is determined by $\chi^{2}$ exact test.

A

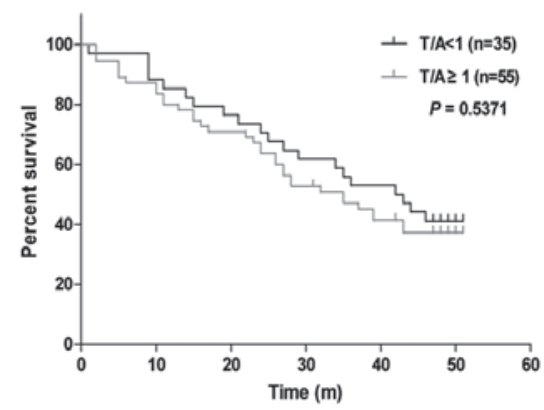

B

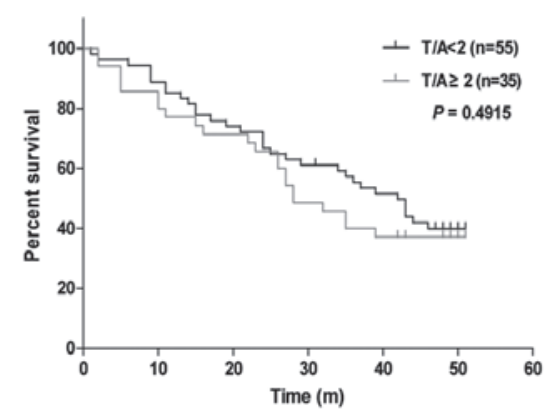

c

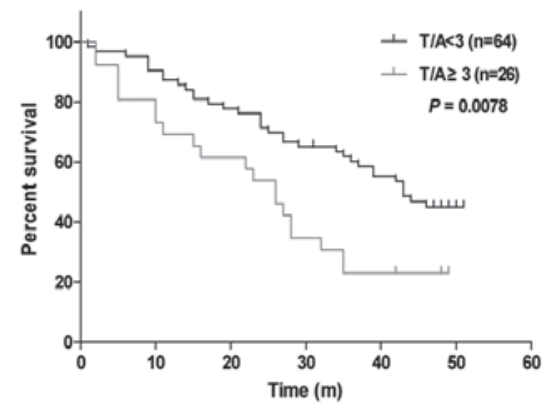

Figure 3. The highest level of FAS overexpression is associated with poor survival of patients with gastric carcinoma. (A-C) show survival curves for patients with carcinomas classified as $\mathrm{S}$ (T/A) $\geq 1, \geq 2$ and $\geq 3$, respectively. FAS overexpression corresponding to $\mathrm{S}$ (T/A) $\geq 1$ or $\geq 2$ was not associated with survival. Patients with $\mathrm{S}$ (T/A) $\geq 3$ had significantly worse overall survival compared with all other patients (log-rank test, $\mathrm{P}=0.0078)$.

several components in these two pathways (4,24). Surprisingly, FAS overexpression was not significantly correlated with total, cytoplasmic or nuclear loss of PTEN (Table II) or pERK expression (data not shown) in gastric carcinomas.

Survival analysis. Finally, we conducted survival analysis to determine whether FAS overexpression in tumors was associated with survival by plotting Kaplan-Meier survival curves and calculating the 3-year survival rate for all three grades of FAS overexpression. For cases with $\mathrm{S}(\mathrm{T} / \mathrm{A}) \geq 1$ or $\geq 2$, FAS overexpression was not associated with overall survival or 3-year survival rate (Table III; Fig. 3A and B). However, among cases with $\mathrm{S}$ (T/A) $\geq 3$, FAS overexpression was significantly associated with poor survival (Fig. 3C, log-rank test, $\mathrm{P}=0.0078$ ) and with a decreased 3-year survival rate (Table III; $\chi^{2}$ test, $\mathrm{P}=0.0023$ ).

\section{Discussion}

FAS protein, also known as oncogenic antigen 519 (OA-519), is overexpressed and hyperactivated in the majority of human malignancies. It plays a central role in the maintenance of the malignant phenotype by enhancing cancer cell survival and proliferation (3). Overexpression of FAS has been reported in human carcinomas including prostate, ovary, breast, colon, endometrium, thyroid gland, squamous cell carcinoma of the lung, and gastric carcinomas. In this study, FAS overexpression was detected in $22.2 \%$ (20/90), $10 \%$ (9/90) and $28.9 \%(26 / 90)$ of the carcinoma tissue specimens graded as $\mathrm{S}(\mathrm{T} / \mathrm{A}) \geq 1$ to $<2$, $\mathrm{S}(\mathrm{T} / \mathrm{A}) \geq 2$ to $<3$ and $\mathrm{S}(\mathrm{T} / \mathrm{A}) \geq 3$, respectively.
Many studies have revealed that FAS overexpression and hyperactivity is regulated by the MAPK/ERK1/2 and PTEN/PI3K/AKT signaling pathways. Therefore, we examined PTEN and pERK expression levels in the same TMA samples. However, FAS overexpression was not correlated with pERK expression or the loss of PTEN in these gastric carcinoma specimens. These findings suggest that there are signaling pathways independent of the PTEN/PI3K/AKT and RAS/RAF/MEK/ERK pathways, such as the ubiquitin-protease pathway, that regulate FAS expression (25).

Although FAS overexpression at any of the three levels defined in this study was not associated with any of the clinicopathological parameters assessed (age, gender, AJCC stage and histological grade), high FAS overexpression [S (T/A) $\geq 3$ ] was significantly associated with poor survival and with a reduced 3 -year survival rate. Although several studies have reported that FAS is overexpressed in gastric cancer $(21,22)$, to our knowledge, our study is the first to show that high FAS overexpression could be a prognostic marker in gastric carcinoma.

In addition to its potential utility as a prognostic marker for cancer patients, FAS shows some promise as a chemotherapeutic target (3,26-28). For example, several studies have shown that cerulenin, a specific noncompetitive inhibitor of the $\beta$-ketoacyl synthase activity of FAS, is selectively cytotoxic to breast and ovarian cancer cells exhibiting enhanced fatty acid synthesis, but not to normal cells with constitutively low FAS expression (29,30). Based on these and other results, more research into the clinical relevance of FAS overexpression is necessary, particularly because FAS may represent an excellent target for treating gastric carcinoma, and other tumors. 


\section{References}

1. Kuhajda FP: Fatty-acid synthase and human cancer: new perspectives on its role in tumor biology. Nutrition 16: 202-208, 2000.

2. Takahiro T, Shinichi K and Toshimitsu S: Expression of fatty acid synthase as a prognostic indicator in soft tissue sarcomas. Clin Cancer Res 9: 2204-2212, 2003.

3. Menendez JA and Lupu R: Fatty acid synthase and the lipogenic phenotype in cancer pathogenesis. Nat Rev Cancer 7: 763-777, 2007.

4. Van de Sande T, De SE, Heyns W, Verhoeven G and Swinnen JV: Role of the phosphatidylinositol 3'-kinase/PTEN/Akt kinase pathway in the overexpression of fatty acid synthase in LNCaP prostate cancer cells. Cancer Res 62: 642-646, 2002.

5. Escot C, Joyeux C, Mathieu M, Maudelonde T, Pages A, Rochefort $\mathrm{H}$ and Chalbos D: Regulation of fatty acid synthetase ribonucleic acid in the human endometrium during the menstrual cycle. J Clin Endocrinol Metab 70: 1319-1324, 1990.

6. Rooney SA: Fatty acid biosynthesis in developing fetal lung. Am J Physiol 257: L195-L201, 1989.

7. Joyeux C, Chalbos D and Rochefort H: Effects of progestins and menstrual cycle on fatty acid synthetase and progesterone receptor in human mammary glands. J Clin Endocrinol Metab 70: 1438-1444, 1990.

8. Smith S and Ryan P: Asynchronous appearance of two enzymes concerned with medium chain fatty acid synthesis in developing rat mammary gland. J Biol Chem 254: 8932-8936, 1979.

9. Chirala SS, Chang H, Matzuk M, et al: Fatty acid synthesis is essential in embryonic development: fatty acid synthase null mutants and most of the heterozygotes die in utero. Proc Natl Acad Sci USA 100: 6358-6363, 2003.

10. Alo PL, Visca P, Trombetta G, et al: Fatty acid synthase (FAS) predictive strength in poorly differentiated early breast carcinomas. Tumori 85: 35-40, 1999.

11. Shurbaji MS, Pasternack GR and Kuhajda FP: Expression of haptoglobin-related protein in primary and metastatic breast cancers. A longitudinal study of 48 fatal tumors. Am J Clin Pathol 96: 238-242, 1991

12. Nguyen PL, Ma J, Chavarro JE, et al: Fatty acid synthase polymorphisms, tumor expression, body mass index, prostate cancer risk, and survival. J Clin Oncol 28: 3958-3964, 2010.

13. Shurbaji MS, Kuhajda FP, Pasternack GR and Thurmond TS: Expression of oncogenic antigen 519 (OA-519) in prostate cancer is a potential prognostic indicator. Am J Clin Pathol 97: 686-691, 1992.

14. Gansler TS, Hardman W III, Hunt DA, Schaffel S and Hennigar RA: Increased expression of fatty acid synthase (OA-519) in ovarian neoplasms predicts shorter survival. Hum Pathol 28: 686-692, 1997.

15. Rashid A, Pizer ES, Moga M, et al: Elevated expression of fatty acid synthase and fatty acid synthetic activity in colorectal neoplasia. Am J Pathol 150: 201-208, 1997.

16. Uddin S, Hussain AR, Ahmed M, et al: High prevalence of fatty acid synthase expression in colorectal cancers in Middle Eastern patients and its potential role as a therapeutic target. Am J Gastroenterol 104: 1790-1801, 2009.
17. Epstein JI, Carmichael M and Partin AW: OA-519 (fatty acid synthase) as an independent predictor of pathologic state in adenocarcinoma of the prostate. Urology 45: 81-86, 1995.

18. De SE, Brusselmans K, Heyns W, Verhoeven G and Swinnen JV: RNA interference-mediated silencing of the fatty acid synthase gene attenuates growth and induces morphological changes and apoptosis of LNCaP prostate cancer cells. Cancer Res 63: 3799-3804, 2003.

19. Menendez JA, Colomer R and Lupu R: Inhibition of tumorassociated fatty acid synthase activity enhances vinorelbine (Navelbine)-induced cytotoxicity and apoptotic cell death in human breast cancer cells. Oncol Rep 12: 411-422, 2004.

20. Menendez JA, Vellon L, Colomer R and Lupu R: Pharmacological and small interference RNA-mediated inhibition of breast cancer-associated fatty acid synthase (oncogenic antigen-519) synergistically enhances Taxol (paclitaxel)-induced cytotoxicity. Int J Cancer 115: 19-35, 2005.

21. Hashimoto T, Kusakabe T, Sugino T, et al: Expression of hearttype fatty acid-binding protein in human gastric carcinoma and its association with tumor aggressiveness, metastasis and poor prognosis. Pathobiology 71: 267-273, 2004.

22. Kusakabe T, Nashimoto A, Honma K and Suzuki T: Fatty acid synthase is highly expressed in carcinoma, adenoma and in regenerative epithelium and intestinal metaplasia of the stomach. Histopathology 40: 71-79, 2002.

23. Enzinger FM and Weiss SW: Soft Tissue Tumors. 3rd edition. Mosby-Year Book, Inc., St. Louis, MO, pp4-12, 1995.

24. Bandyopadhyay S, Pai SK, Watabe M, et al: FAS expression inversely correlates with PTEN level in prostate cancer and a PI 3-kinase inhibitor synergizes with FAS siRNA to induce apoptosis. Oncogene 24: 5389-5395, 2005.

25. Graner E, Tang D, Rossi S, et al: The isopeptidase USP2a regulates the stability of fatty acid synthase in prostate cancer. Cancer Cell 5: 253-261, 2004

26. Flavin R, Peluso S, Nguyen PL and Loda M: Fatty acid synthase as a potential therapeutic target in cancer. Future Oncol 6: 551-562, 2010.

27. Kridel SJ, Lowther WT and Pemble CW: Fatty acid synthase inhibitors: new directions for oncology. Expert Opin Investig Drugs 16: 1817-1829, 2007.

28. Kuhajda FP: Fatty acid synthase and cancer: new application of an old pathway. Cancer Res 66: 5977-5980, 2006.

29. Pizer ES, Wood FD, Heine HS, Romantsev FE, Pasternack GR and Kuhajda FP: Inhibition of fatty acid synthesis delays disease progression in a xenograft model of ovarian cancer. Cancer Res 56: 1189-1193, 1996.

30. Pizer ES, Jackisch C, Wood FD, Pasternack GR, Davidson NE and Kuhajda FP: Inhibition of fatty acid synthesis induces programmed cell death in human breast cancer cells. Cancer Res 56: $2745-2747,1996$ 